\title{
Automation, Architecture and Labour
}

Mollie Claypool

Increasing automation over the last decade has transformed much of the way we interact, communicate and produce work as architects and designers. Platforms, connecting us through the power of computation and the Internet of Things - and the economic model for automation - have proliferated into our daily lives. We are reliant on these platforms. When the internet goes down or when a software app won't update, so does our capacity to participate in the economy, to produce and reproduce. I see my internet extender flicker. Where I live in Bristol, United Kingdom is the worst place in the country for broadband blackouts. ${ }^{1}$

I am writing this from a mid-terraced house that was built in 1886 out of stone and brick, in a neighbourhood used previously for coal and clay mines. This area has the worst pollution in the city, with $11 \%$ of premature deaths caused by air pollution. ${ }^{2}$ Yet house prices have gone up $120 \%$ percent here in the last decade. ${ }^{3}$ Affordability has continued to worsen, with some people in England expected to spend fifteen times their annual earnings to buy a home. ${ }^{4}$ In England alone over 8.4 million people are affected by this crisis in housing, with overcrowding, hidden households, unsuitable or poor quality housing, homelessness and unaffordability affecting 1 in 7 people. ${ }^{5}$ Yet state capital spent on housing has decreased by $50 \%$ in the last 20 years. ${ }^{6}$ Lack of vision, minimal investment and deregulation by the state since the 1970s has left the sector extremely susceptible to economic actors that see an opportunity to make a profit. And this is not a local condition. Worldwide, over 2 billion homes need to be built in the better part of the next century. ${ }^{7}$

But in addition to widening failures in governance and policy that have constructed rising inequities in housing such as the lack of meaningful investment in building council housing, ${ }^{8}$ the architecture and construction industries are also not well-situated to deliver on these needs. Architecture is highly marginalised, with a report completed for the Architect's Council of Europe finding in 2019 "that there is

\footnotetext{
${ }^{1}$ Mark Sweney, "Bristol is the worst UK city for broadband outages with 169 hours a year", The Guardian, $<$ https://www.theguardian.com/technology/2020/aug/13/bristol-is-worst-uk-city-for-broadband-outages-with-169hours-a-year>, August 132020 (accessed 14 August 2020).

2 J. H. Barnes, T. J. Chatterton, "An Environmental Justice Analysis Of Exposure To Traffic-related Pollutants In England And Wales", WIT Transactions on Ecology and the Environment, 210: 431-432, 2017.

${ }^{3}$ Miles Brignall, "Where have UK house prices increased most - and least - since 2010", The Guardian, https://www.theguardian.com/money/2020/oct/10/where-have-uk-house-prices-increased-most-and-least-since2010, October 10, 2020 (accessed 10 October 2020).

4 "Housing affordability in England and Wales: 2019", Office for National Statistics, 2019.

5 " 1 in 7 people in England directly hit by the housing crisis", National

Federation, <https://www.housing.org.uk/news-and-blogs/news/1-in-7-people-in-england-directly-hit-by-thehousing-crisis/>, 2019, accessed 20 September 2020.

6 "The story of social housing", Shelter, <https://england.shelter.org.uk/support_us/campaigns/story_of_social_housing>, accessed 10 September 2020.

${ }^{7}$ Sean Smith, "The world needs to build 2 billion new homes over the next 80 years", World Economic Forum, $<$ https://www.weforum.org/agenda/2018/03/the-world-needs-to-build-more-than-two-billion-new-homes-over-thenext-80-years>, March 02 2018, accessed 20 September 2020.

8 "The story of social housing", Shelter, <https://england.shelter.org.uk/support_us/campaigns/story_of_social_housing>, accessed 10 September 2020.
} 
almost no convincing evidence of the value of architects. ${ }^{\prime 9}$ Construction is a highly striated industry that suffers from a significant labour shortage ${ }^{10}$ is one of the least digitised industries worldwide (second only to hunting) ${ }^{11}$ and productivity has flatlined since the middle of the 20th century. ${ }^{12}$ This sits in stark contrast to other industries that have been able to adopt (or emerge due to) digitisation, where growth and productivity has skyrocketed. And an increasing wealth divide has emerged. Capital is more and more centralised with the richest $1 \%$ owning $44 \%$ of the world's wealth. ${ }^{13}$ It is not a coincidence that this divide runs in parallel to increasing automation in most industries.

And so it is this intersection between the distribution of automation, capital, activism, housing and people that the work we (myself, Gilles Retsin and Manuel Jimenez Garcia) do with our team (Kevin Saey, Clara Jaschke, Nikolaos Tsikinis, Tomas Tvarijonas, Danai Parissi and David Doria) in our practice Automated Architecture Ltd (AUAR) and research laboratory AUAR Labs at The Bartlett School of Architecture, UCL is situated. In this short essay, I will provide the broader context for the work of AUAR and design research of AUAR Labs. Originally grounded in experimental design speculations by our students in both MArch Unit 19 in the MArch Architecture programme from 2012-2018 and Research Cluster 4 (RC4) (where I teach theory) in the post-graduate MArch Architectural Design programme from 2015 onwards, the work our AUAR/AUAR Labs has shifted this work outside of the realm of academia into working directly with the communities most affected by the increasing financialisation of housing and automation.

\section{AUTOMATION AS A DESIGN PROJECT}

For decades, the dominant narrative around automation in construction has been around the loss of jobs using manual labour. Often the 'digital fabrication' technologies that are developed to replace these jobs focus purely on the productivity gained by implementing the use of a single-task robot: a bricklaying robot, ${ }^{14}$ a tile laying robot, a welding robot, etc. Increases in productivity equals increased wealth for the owners of these technologies. Unfortunately these robots require significant investment only possible with the backing of venture capital. These kinds of robots are products only available for those with extreme wealth to begin with - when launched in 2015 SAM the bricklaying robot by Construction Robotics cost half a million US dollars. ${ }^{15}$ And yet these robots are extremely limited -

\footnotetext{
${ }^{9}$ Rosalie Callway, Lorraine Farrelly, Flora Samuel, "The Value of Design and the Role of Architects: A study for ACE Architect's Council of Europe prepared by the School of Architecture University of Reading", $<$ https://www.ace-cae.eu/uploads/tx_jidocumentsview/Value_of_Design.pdf>, March 2019, accessed 10 October 2020.

${ }^{10}$ S. MacKenzie,A. R. Kilpatrick \&A. Akintoye, "UK construction skills shortage response strategies and an analysis of industry perceptions", Construction Management and Economics, 18: 852-852, 2000.

${ }^{11}$ Rajat Agarwal, Shankar Chandrasekaran, and Mukund Sridhar, "Imagining construction's digital future", McKinsey Global Institute, <https://www.mckinsey.com/business-functions/operations/our-insights/imaginingconstructions-digital-future>, June 24,2016, accessed 10 October 2020.

12 Ibid.

13 "Global Inequality", Inequality Org, <https://inequality.org/facts/global-inequality/>, accessed 8 August 2020.

14 "SAM 100", Construction Robotics, <https://www.construction-robotics.com/sam100/>, accessed 8 August 2019.

15 "Bricklaying robot SAM - on your site for $£ 330,000$ ", Construction Manager, $<$ https://www.constructionmanagermagazine.com/s1am-bri1ck-laying-rob2ot-price-tag/> 04 September 2015, accessed 8 August 2019.
} 
while they are technical innovations in 'digital fabrication' or 'digital construction', they are solving very narrow problems.

Investment in construction automation has also occurred at the scale of the factory. In 2019 Katerra, a California-based country creating modular building systems primarily for housing, became the first Unicorn company in construction. Taking a similar approach to automation as automobile manufacturing, modular housing factories such as Katerra results in the centralisation of housing production to off-site factories, disconnected to the contexts in which these systems are deployed. Furthermore, the workers that build the housing systems that companies like Katerra produce are currently 'imported' onto sites from elsewhere, resulting in the marginalisation of local employment and economic development opportunities. Factory-made housing is seen as displacing local jobs, which creates resistance and scepticism among local communities and creates limited localised capacity for housing produced using automation. ${ }^{16}$

A critique of both this narrow definition of a robot, as well as the centralisation of automation that disables access to technology by local communities and contexts can be connected to a critique of neoliberal capitalism in architectural production. Automation only in the terms of 'digital design' and 'digital fabrication' are what Nick Srncek and Alex Williams refer to as 'folk politics'. Folk politics are "tactics and strategies which were previously capable of transforming collective power into emancipatory gains" but are now so divorced from actual mechanisms of power that they are "incapable of transforming capitalism" and have become "drained of their effectiveness." 17 'Digital design' and 'digital fabrication' - 'digital X' - have been long heralded within the discipline as being transformative.

Yet by being focused on solving small scale problems within the discipline, 'digital design' - and its focus on representation, variation and affect - and 'digital fabrication' - and its focus on replicating craft - effectively enables collective disempowerment and marginalisation of architects within the discourse on automation. Other actors, such as those with capital to develop and implement automation, and therefore economically benefit from it, have emerged as a result of the continued acceptance of the transformative promise of the digital made almost 30 years ago, despite evidence pointing in the opposite direction: towards the failure of the digital to radically democratise production.

The 'way things are done' within architecture is not just accidental. Disciplinary practices are historically constructed. ${ }^{18}$ In the last several decades, these disciplinary practices benefit neoliberalism, which suppresses the strength and power of the local or small-scale, effectively dismantling the power of 'bottom up' change (hence resulting in folk politics). And as architects have become more marginalised, they too have become small-scale actors, and thus increasingly suppressed. To broaden the scope of automation in architecture from the discourse around the 'digital $X$ ' or historical disciplinary practices and towards automation is emancipatory, both for the practice of architecture as

\footnotetext{
${ }^{16}$ See Melissa Mean, Craig White, \& Eleanor Lasota, We Can Make: Civic Innovation in Housing, We Can Make..., 2017.

${ }_{17}$ Nick Srnicek and Alex Williams, Inventing the Future: Postcapitalism and a World Without Work, Verso, 2015, p 10.

18 lbid.
} 
well as the people that it should be serving. Architecture in the age of automation can no longer ignore that it too must change.

Design operates not just at the scale of the technological: it is social, it is economic. Design is politics. Architects can either contribute to changing notions of the role and value of design, or succumb to greater irrelevance. So core to the work of AUAR/AUAR Labs is the notion that automation is a design project. ${ }^{19}$ When framed as a design project, automation becomes an arena through which architects can raise and discuss issues such as ownership, distribution, labour and the culture and impact of automation on architectural production. These are shared and global issues that transcend place, cultures and contexts. By addressing them through automation, architects can understand the design of automation in architectural production as a collective social project.

Architects can also design a collective dvision for automation. Perhaps, as the political theory of accellerationism argues, capitalism can be repurposed, by "preserv[ing] the gains of late capitalism while going further than its value system, governance structures, and mass pathologies will allow." 20 This vision can therefore be one that does not centralise or monopolise automation as neoliberalism does, but demonstrates the capacity for automation to accelerate the production of distributed abundance, and amplify interdependence, ${ }^{21}$ instead of enabling further austerity, divisiveness and marginalisation. A vision for automation in architecture must: confront the value systems and hierarchies embedded in both architecture's social and spatial practices by radically rethinking architectural syntax in terms of geometry, tectonics and aesthetics; redefine automation's capacity to enable increasing access to architectural production for the benefit of the many, rather than the few; and consider automation as multi-scalar - as both 'on the ground' tech for design production and as a framework for coordination and logistics across scales and contexts.

\section{ARCHITECTURE FOR AUTOMATION}

\section{Insert Figure}

This requires designing architecture for automation. To do this is to go to the core of architecture, which for thousands of years has been designed in relationship to localised contexts and resources. Yet increasing globalisation since World War II has disrupted this tradition: a building can be designed in New York, its parts manufactured in Beijing, Germany or Shanghai and be built in Cairo or Hong Kong or Johannesburg. It is now evident that this practice is unsustainable, with the building and construction industry contributing to $39 \%$ of all carbon emissions. ${ }^{22}$ Insert Figure

Thirty years of 'digital design' has further contributed to this, enabling architects to design complex and intricate geometries. These buildings have thousands of bespoke, one-off, parts costly to manufacture

\footnotetext{
${ }^{19}$ Mollie Claypool, Manuel Jimenez Garcia, Gilles Retsin and Vicente Soler, Robotic Building: Architecture in the Age of Automation, Detail Edition, 2019.

${ }^{20}$ Nick Srnicek and Alex Williams, "\#Accelerate Manifesto for an Accelerationist Politics," Critical Legal Thinking, ( $<$ http://criticallegalthinking.com/2013/05/14/accelerate-manifesto-for-an-accelerationist-politics/ >, 2013, accessed 9 September 2020.

${ }^{21}$ This term can be credited to Amahra Spence, creative director of MAIA Group (Birmingham, UK), who used it to describe a core goal of MAIA's work at the event Making Together: Exploring New Tech, Tools and Tactics to Level Up Communities at Bristol Housing Festival, October 2020.

22 https://www.worldgbc.org/sites/default/files/WorldGBC Bringing Embodied Carbon Upfront.pdf $\mathrm{p} 7$
} 
in a highly striated and inefficient production chain. This is not at all a kind of architecture that can speak to automation, which requires similarity, repetition and seriality. A new generation of 'digital architects' and designers have confronted this contradiction by radically rethinking the very parts that make up buildings. This is done utilising the concept of the Discrete. ${ }^{23}$

\section{Insert Figure}

The Discrete builds on the notion of digital materials, or a new kind of building block "assembled from a discrete set of parts, reversibly joined in a discrete set of relative positions and orientations" that has the same structure as data in a computer programme. These new, wholly digital building blocks can then be organised into different positions, which in principle can be continuously altered. ${ }^{24}$ This dramatically reduces the number of different kinds of parts that make up a building. The same or similar building blocks able to creates part-to-whole ${ }^{25}$ relationships that enables patterns ${ }^{26}$ or assemblies to emerge from these building blocks being combined together to serve different architectural functions. Staircases, beams, slabs, columns all can be designed using a single block.

\section{Insert Figure Voxel Chair} Insert Figure INT

A Discrete architectural syntax can act as a universal framework for architectural production, supporting diversity of geometries, forms and tectonics to emerge in response to particular contexts. It is also an architectural syntax well-suited for increasing automation: 3D printing, robotic assembly or other digital fabrication methods such as CNC machines can be used by a design project using this thinking. The Discrete also shortens the production chain for buildings without increasing costs as the same part can be used to form the entirety of a building's structure and spatial configurations.

\section{Insert Semblr}

\section{Insert ALIS}

It is within this context that projects such as Semblr by Ivo Tedbury (2017) and Automated Living System (ALIS) by Akhmet Khakimov, Estefania Barrios, Evgenia Krassakopoulou, Joana Correia, Kevin Saey (2019), student work in Unit 19 and RC4, have been developed. Each of the projects developed a single, repeatable building block that could be manufactured using a common digital fabrication technology like a CNC machine. This also presents the challenge of developing a Discrete building block that creates minimal waste: each project uses only a single sheet of plywood to form one block, sitting in direct contrast to earlier 'digital' work where variation in size of parts results in significant material waste.

Insert Semblr sheet

Insert ALIS sheet

\section{AUTOMATION FOR ASSEMBLY}

\footnotetext{
${ }^{23}$ For an overview of the architects and designers working on this topic, see Gilles Retsin (ed.), Architectural Design, 89, 2, Wiley \& Sons, 2019.

${ }^{24}$ Neil Gershenfeld, Matthew Carney, Benjamin Jenett, Sam Calisch, and Spencer Wilson, "Macrofabrication with Digital Materials: Robotic Assembly," Architectural Design, 85, 5, Wiley \& Sons, 2015.

25 Daniel Koehler, The Mereological City: a reading of the works of Ludwig Hilberseimer, Transcript, 2016.

26 Jose Sanchez, Architecture for the Commons: Participatory Systems in the Age of Platforms, Routledge: 80-82, 2020.
} 


\section{Insert Figure Semblr}

Part-to-whole relations in a Discrete framework also enables automation to be used at the scale of the building assembly, distributing the labour it takes to construct a building away from the manual labour of construction workers and into robotic automation. I have previously called this approach Discrete Automation. ${ }^{27}$ This also throws into question the notion of ownership of space: could housing be shared, used and inhabited in new ways due to automation? In ALIS, Semblr, Pizzabots by Mengyu Huang, Dafni Katrkakalidi, Martha Masli, Man Nguyen and Wenji Zhang (2019) and MOBO by Pofu Yang, Nadia Saki, MengMeng Zhao, Tian Chuan and Keshia Lim (RC4, 2019), the potential of modular robotic assembly was explored to begin to answer this question.

\section{Insert Figure Semblr \\ Insert Figure Pizzabot}

Tedbury developed a distributed modular robot capable of distributing the building blocks, enabling homes to be created quickly with minimal human intervention. He has extended this work postgraduation from Unit 19 in the startup company Semblr, further developing this work into novel modular bricklaying robots. ${ }^{28}$ The distributed modular robots developed for ALIS more closely mimicked the geometry of the building block itself. Spaces unused in an apartment building during a typical work day were 'activated' by these robots, with the building to change and adapt throughout the day to changing needs of its inhabitants, determined via a mobile app. This allowed all space available within a building to be used - whether it was being used for work or rest - through ongoing robotic assembly and disassembly of different spatial configurations. Pizzabots almost entirely blurred the boundaries between what was 'building' and what was 'automation', designing a robot the exact geometry of the building block itself (the size of a pizza box), enabling the robot to entirely merge with, and negotiate, the configuration and reconfiguration of the building. In $\mathrm{MOBO}$, the robot was made of modular parts that each carry out a single type of movement and can be combined in different ways, resulting in an ecology of construction robots fit for a variety of different assembly tasks.

Insert Figure Mobo robot

This work sits in direct relationship to the work of other researchers such as Maria Yablonina, who envisions small robots working alongside workers that are "continuously performing construction and spatial reconfiguration tasks in response to their human co-habitants." ${ }^{29}$ This raises crucial questions around ownership, decision-making and data, and has significant ecological implications as building blocks can be continuously re-used into other spatial assemblies. Yet these projects also present a more speculative approach to automation: we are not yet quite in a world where distributed modular robots will be on building sites, or 'living' and 'working' amongst people in buildings, negotiated through an app. Our assumptions about what a home is sits in opposition to the temporality of Discrete Automation. So how can automation be implemented in architecture today? How can communities access automation without full automation? How can new understandings about home and place emerge? A possible answer lies in platforms, the economic model of automation.

\footnotetext{
${ }^{27}$ Mollie Claypool, "Discrete Automation", e-flux architecture, <https://www.e-flux.com/architecture/becomingdigital/248060/discrete-automation/> 2019, accessed 1 August 2020.

${ }^{28}$ Link to Semblr

29 SPACE10
} 


\section{PLATFORMS AS COLLECTIVE RESILIENCE}

The distribution of automation into communities requires, as argued by Nick Srnicek in Platform Capitalism (2017) that the rise of platforms within capitalism (Facebook, AirBnB, Uber) are understood not as cultural actors but as extractive economic actors, out to make the most profit from citizens that use their platforms. As such, these companies are seen not as creating platforms for the common 'good', but in the pursuit of power, extraction, and wealth. ${ }^{30}$ Srnicek also predicts that due to the rising inequalities produced by these platforms, eventually, platforms as a business model will fail. His solution is to suggest the notion of the 'public platform'. ${ }^{31}$

The work of students in Unit 19 and RC4 have explored the public platform through the development of mobile apps owned by cooperative housing companies for connecting people to housing continuously adapted by distributed robots in response to the communities changing needs. The work of AUAR/AUAR Labs has focused on the public platform at three scales: (1) a Discrete building system called Block Type $A$ that anticipates increasing automation while reducing the threshold to access by local communities; (2) co-designed and values-centred ${ }^{32}$ software platform that enables people to access Block Type from design to assembly/reassembly; and (3) on increasing the capacity for digital labour within local communities that are building their own homes.

\section{Insert Figure}

A development of the CNC'd building blocks from ALIS, Block Type A is a Discrete building system for housing using a timber block that is post-tensioned locally to achieve global stability in larger assemblies. Through distribution of both the skills, tools and technologies to make Block Type A into communities, the system sits in opposition to a centralised, off-site factory model. The design of the block significantly reduces the access threshold for community members, with minimal training and no specialist tools required for prefabrication or assembly. Block Type A in particular is well-suited for full robotic prefabrication, and anticipates this as our projects build up technological infrastructure in the communities we are working.

AUAR has developed an ecology of apps for using Block Type A and other Discrete building systems under development. These tools form the base technologies of a platform, as they link together design, fabrication and assembly in accessible ways. Co-designed with local communities as well as trades and craftspeople, the software tools have taken a values-centred approach: putting communities that want to use these tools at the centre of the tool development. The community member's expertise and lived experience directly informs the capacity of the apps, providing instructive, game-like environments that empower the community to design, fabricate and build the homes they need.

\section{Insert Figure}

In the recent project Block West (2020) designed over a 6 month period in Knowle West, Bristol, 25 community members ages 12 to 76 participated in the prefabrication and assembly of a housing and community space prototype using 145 Block Type A over a 10 day period. The project provided parttime jobs in the local community throughout the project, serving as a prototype for community

\footnotetext{
${ }^{30}$ Nick Srnicek, Platform Capitalism (Polity, 2017).

$31 \mathrm{lbid}, 128$.

${ }^{32}$ Add
} 
engagement, localised investment, and pilot digital construction skills training programme for AUAR Labs partners' Knowle West Media Centre's We Can Make homes programme, a community-led housing project ongoing in Knowle West that is about to break ground for their first 2 homes. This is important in an area like Knowle West where there is no centre or community hub and where there has been historically very little investment. It is often a place where money often "washes through" rather than adding sustainable value or social infrastructure.

\section{AUTOMATION AS NEW CITIZENSHIP}

The distribution of skills, knowledge and expertise about Discrete Automation within local communities has served as a first stage of platform development, where citizens are activated in new ways to take ownership of their community and built environment. Digital labour becomes about investing in local skills and knowledge, levelling up communities to further scale and coordinate efforts across the community and beyond. As a Block West participant said, "We are literally building the community from the bottom up. [The prototype] I've helped make isn't mine. It's ours. That's the most important thing to come out of this - it's given me ownership of my community. It's giving people different choices, better choices about how things can be. And it feels like only the beginning."

This work aims to create collective resilience within the communities most at risk of the 'status quo' of automation in housing production: materially, technologically and socially. As a process, projects like ALIS, Semblr and other student work serve as a catalyst for projects like Block West, demonstrating the speculative vision for automation in housing. AUAR/AUAR Labs projects develop the real, local technological and social infrastructure for these visions to be eventually realised when automation becomes more distributed, affordable and thus more accessible. These projects, by serving as prototypes for AUAR/AUAR Labs, are able to be replicated and adapted into other localised contexts: it is a realised example of how automation can be multi-scalar and values-centred: we are now working in three different communities across the UK using the Discrete framework outlined in this essay. This approach sits at the intersection of communities, research and practice. It also creates novel possibilities around the distribution of automation, capital and housing, enabling new forms of engaged and empowered citizenship to emerge within the communities we are working. This requires an activist-based approach: it is hard, long, but rewarding work. To do this is to embed ourselves within the communities we are working, to gain and build trust and break down disciplinary silos between academia and practice, between communities and 'experts', in order to enact the changes we hope to see in housing and automation.'

\section{References}

J. H. Barnes, T. J. Chatterton, "An Environmental Justice Analysis Of Exposure To Traffic-related Pollutants In England And Wales", WIT Transactions on Ecology and the Environment, 210: 431-432, 2017.

Miles Brignall, "Where have UK house prices increased most - and least - since 2010", The Guardian, https://www.theguardian.com/money/2020/oct/10/where-have-uk-house-prices-increased-most-and-least-since2010, October 10, 2020 (accessed 10 October 2020).

“Housing affordability in England and Wales: 2019”, Office for National Statistics, 2019. 
Mark Sweney, "Bristol is the worst UK city for broadband outages with 169 hours a year", The Guardian, $<$ https://www.theguardian.com/technology/2020/aug/13/bristol-is-worst-uk-city-for-broadband-outages-with-169hours-a-year>, August 132020 (accessed 14 August 2020). 